\title{
Pigmented paravenous retinochoroidal atrophy associated with Vogt-Koyanagi- Harada disease: a case report
}

\author{
Prithvi Ramtohul (D), Alban Comet, Pierre Gascon and Danièle Denis
}

\begin{abstract}
Background: To describe a unique case of pigmented paravenous retinochoroidal atrophy that developed several years after Vogt-Koyanagi-Harada disease.

Case presentation: A 28-year-old woman presented with gradual vision loss in both eyes and nyctalopia for 2 years. Past medical history was relevant for Vogt-Koyanagi-Harada disease since the age of 19 and positive HLADR4. Funduscopic examination revealed perivascular pigmentary clumping and atrophic changes radiating from the optic disks. Spectral domain optical coherence tomography through the macula demonstrated perifoveal outer retinal layers loss with cystic degeneration. Fundus autofluorescence showed zonal areas of hypoautofluorescence corresponding to the areas of atrophy. Full-field electroretinogram identified mildly reduced scotopic and photopic responses. The patient was diagnosed with pigmented paravenous retinochoroidal atrophy.

Conclusions: Pigmented paravenous retinochoroidal atrophy may be acquired after Vogt-Koyanagi-Harada disease. Pathogenesis of pigmented paravenous retinochoroidal atrophy may involve inflammatory-related precursors on a background of genetic predisposition.
\end{abstract}

Keywords: Pigmented paravenous retinochoroidal atrophy, Retinitis pigmentosa, Spectral domain optical coherence tomography, Vogt-Koyanagi-Harada

\section{Background}

First termed retinochoroiditis radiata in 1937, pigmented paravenous retinochoroidal atrophy (PPRCA) is a rare form of chorioretinal atrophy characterized by perivenous retinal pigment epithelial atrophy and pigment clumping [1]. It is typically bilateral, symmetric and non-progressive or slowly progressive and is most often discovered incidentally during routine fundus examination. Vision is generally normal or mildly reduced at presentation, with minimal to no progression over time [2]. Although the etiology of PPRCA remains unknown, there are several hypotheses including genetic, inflammatory or infectious causes. Heterozygous $C R B 1$ variant of uncertain significance in a family with dominantly inherited PPRCA and variable expressivity has been reported [3]. Similarly, tuberculosis, congenital syphilis, Behçet disease, measles and rubella have been proposed as causative [4].

\footnotetext{
* Correspondence: pramtohul@me.com

Centre Hospitalier Universitaire de l'Hôpital Nord, chemin des Bourrely, 13015 Marseille, France
}

We reported a unique case of PPRCA occurring in a background of Vogt-Koyanagi-Harada disease and raised pathophysiologic hypothesis based on optical coherence tomography findings.

\section{Case presentation}

A 28-year-old woman was referred for gradual vision loss in both eyes and nyctalopia for 2 years. Past medical history was relevant for Vogt-Koyanagi-Harada (VKH) disease since the age of 19 and positive HLA-DR4 (Fig. 1). She was treated with systemic corticosteroids administered orally at a dose of $1 \mathrm{mg} / \mathrm{kg} /$ day and azathioprine $(1 \mathrm{mg} / \mathrm{kg} /$ day $)$ for 1 year. No recurrences were observed and the patient was lost to follow-up.

At current presentation, best-corrected visual acuity was 20/25 in both eyes. Anterior segment examination was unremarkable. Funduscopic examination revealed circumscribed areas of retinochoroidal atrophy and pigment clumping along the retinal veins (Fig. 2a). Fundus autofluorescence (AF) showed zonal areas of hypoAF along the 


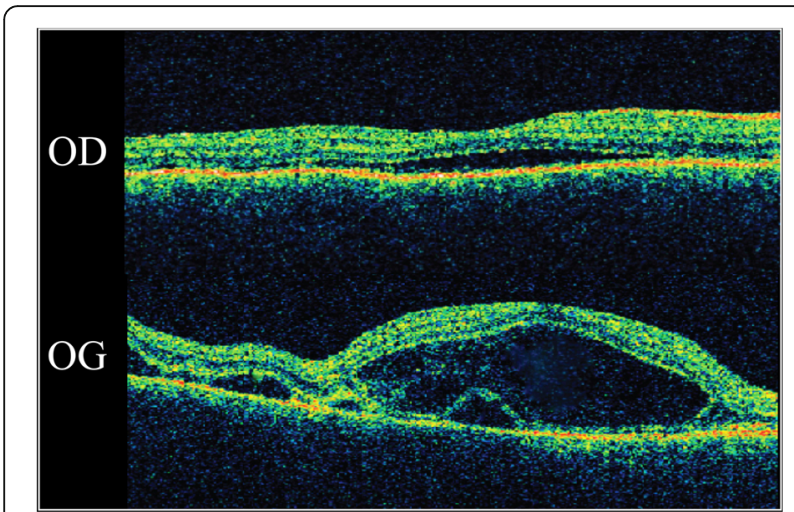

Fig. 1 Initial time domain optical coherence tomography at the age of 19 showed serous retinal detachments with subretinal septa consistent with acute Vogt-Koyanagi-Harada disease retinal veins surrounded by linear hyperAF edges (Fig. 2b). Spectral domain optical coherence tomography (SD-OCT, Spectralis, Heidelberg Engineering, Heidelberg, Germany) through the macula demonstrated perifoveal outer retinal layers disruption with cystic degeneration in both eyes (Fig. 2c). SD-OCT through the atrophic lesions highlighted loss of the outer retinal layers, retinal pigment epithelium (RPE) atrophy, and extensive inner choroidal thinning with preservation of the Haller's layer vessels (Fig. 3a). Perivenular SD-OCT scan revealed thickening of the retinal nerve fiber layer (RNFL) (Fig. 3b). Full-field electroretinogram identified mildly reduced scotopic response and a slight decrease in b-wave amplitude (photopic response). The patient was diagnosed with pigmented paravenous retinochoroidal atrophy.

\section{Discussion and conclusions}

PPRCA is a rare disease characterized by perivenous aggregations of pigment clumping associated with zonal

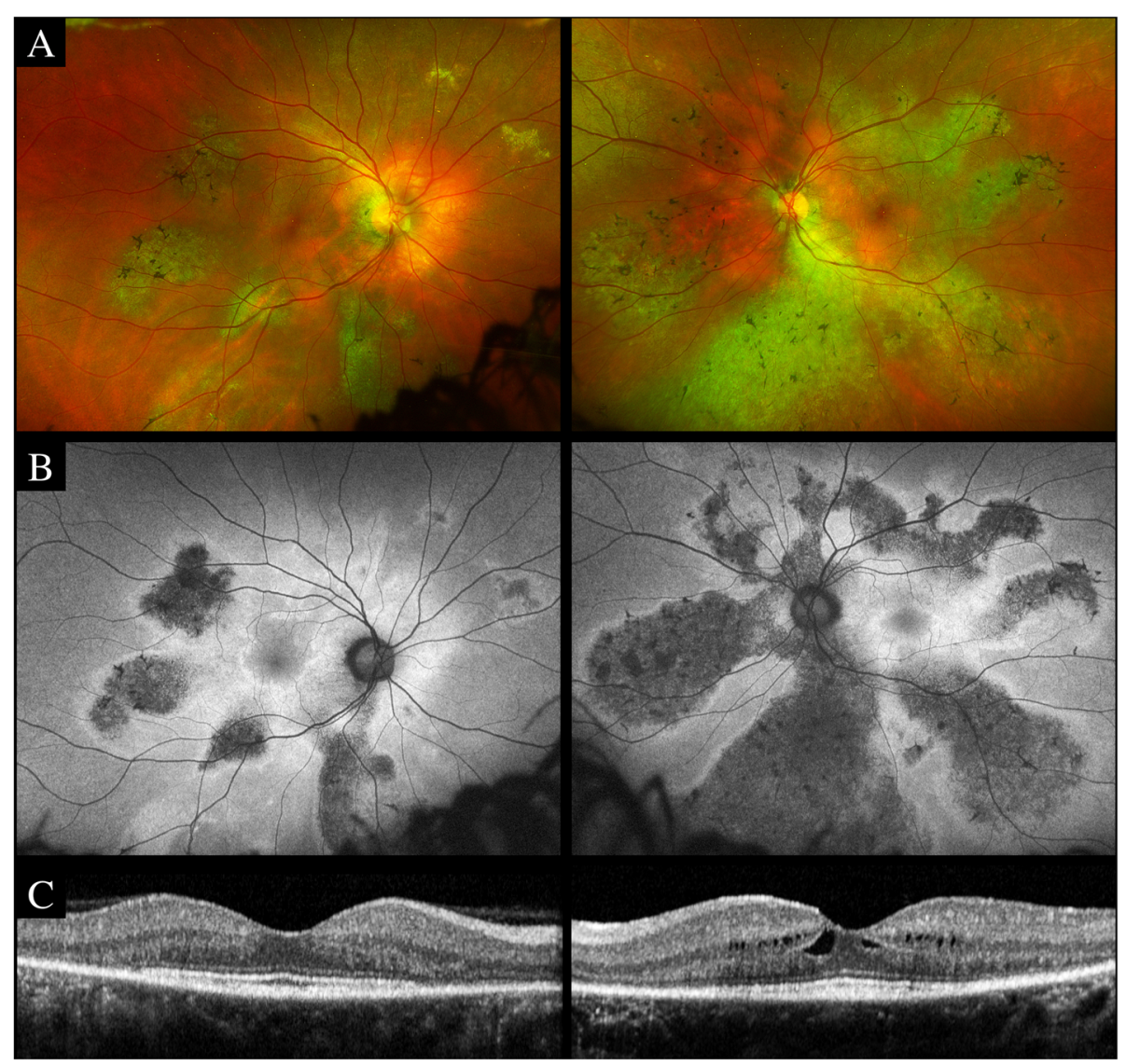

Fig. 2 a. Ultra-widefield fundus photography (Optos, Dunfermline, UK) demonstrated zonal areas of retinochoroidal atrophy distributed along the retinal veins with bone-spicule pigmentation and sparing of the macula. b. Fundus autofluorescence (AF) (Optos, Dunfermline, UK) showed zonal areas of hypoAF along the retinal veins surrounded by linear hyperAF edges. c. Spectral domain optical coherence tomography (Spectralis, Heidelberg Engineering, Heidelberg, Germany) through the macula demonstrated perifoveal outer nuclear layers thinning, ellipsoid and interdigitation zones disruption and cystic degeneration in both eyes 


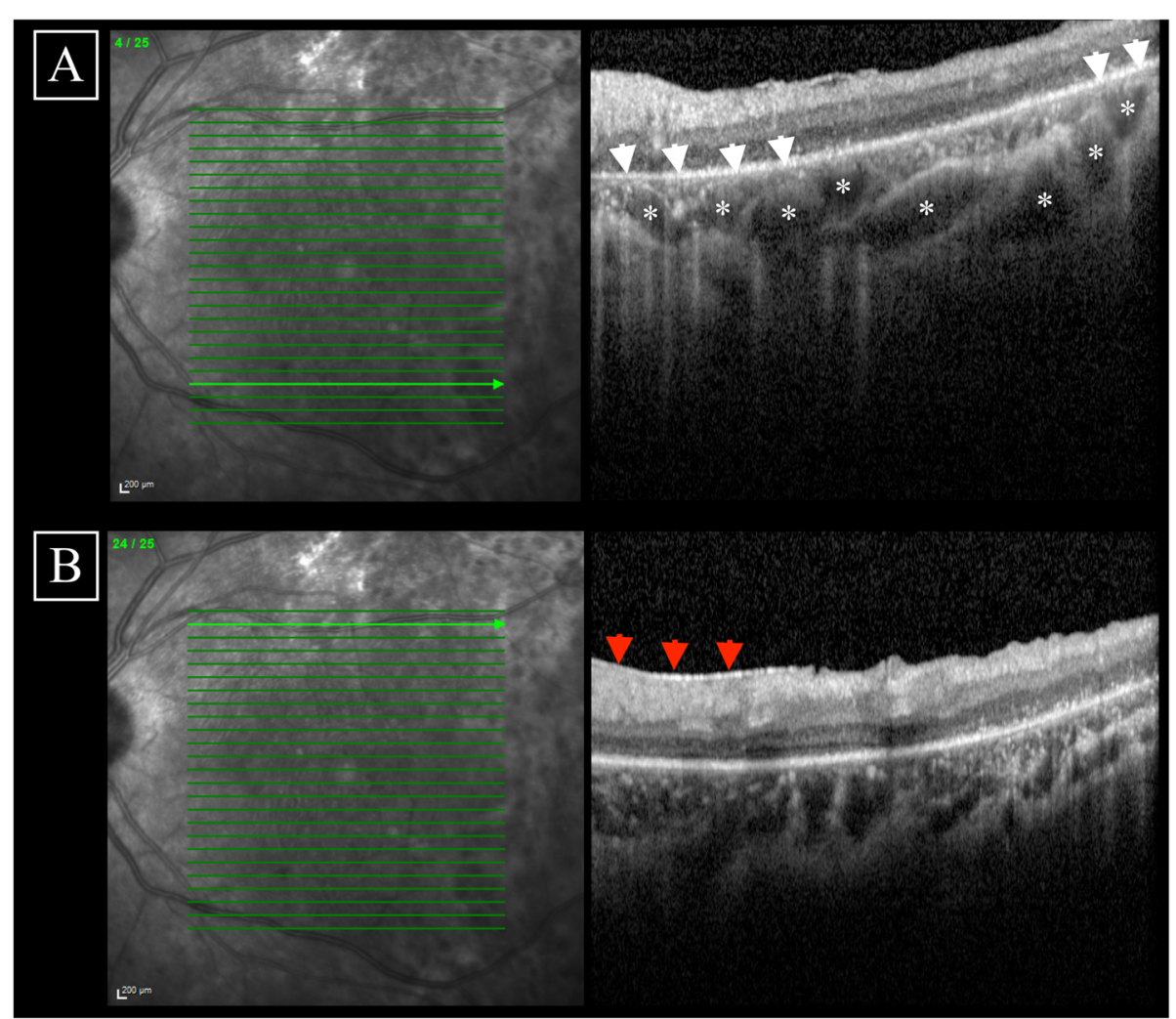

Fig. 3 a. Spectral domain optical coherence tomography (SD-OCT) showed extensive choroidal changes with choriocapillaris and inner choroidal atrophy (red arrowheads) with persistent Haller layer's vessels (asterisks). Note the mark penetration of optical coherence tomography infrared light through the areas of retinal pigment epithelium loss. $\mathbf{b}$. SD-OCT demonstrated thickening and cystic degeneration of the retinal nerve fiber layer (white arrowheads)

areas of retinochoroidal atrophy distributed along the retinal veins. Patients are typically asymptomatic and the disease process is non-progressive or slowly progressive [1]. The underlying etiology of PPRCA remains controversial and a hereditary nature appeared to be a reasonable assumption since McKay et al. reported a dominantly inherited PPRCA in a family with a heterozygous CRB1 variant [3]. Similarly, Obata et al. described PPRCA in two Japanese siblings with variable expressivity [5]. However, in a recent large-cohort study of 23 patients, Shona et al. found no evidence of a genetic basis because $96 \%$ of patients had no familial history of inherited ocular disease [6]. Interestingly, there a several evidence in the literature reporting PPRCA onset after an episode of intraocular inflammation, including tuberculosis, congenital syphilis, Behçet disease, measles and rubella immunization or vaccination [6]. To our knowledge, we report the first case of PPRCA associated with VKH disease and positive HLADR4. This further raises the possibility of triggering inflammatory events on a background of genetic predisposition.

Multimodal imaging techniques have led to better characterization of PPRCA [7]. In our case, geographic areas of RPE loss on SD-OCT corresponded to areas of reduced $\mathrm{AF}$ and thinning of the outer retinal layers colocalized with the linear increased AF. We also documented cystic macular edema (CME) which has exceptionally been described in PPRCA [6]. The authors postulated that the underlying pathogenesis may be comparable to retinitis pigmentosa-associated CME, including blood-retinal barrier breakdown, dysfunction of the RPE pumping function, Müller cells failure and vitreous traction [8]. Intriguingly, SD-OCT scan through the atrophic lesions showed perivenular thickening of the RNFL. This observation has been reported only in one case [9]. This singular SD-OCT feature is of physiopathological interest; in fact, it was hypothesized that choroidal atrophy resulting in decreased choroidal perfusion and subsequent insufficient metabolic supply for the outer retinal layers preceded the photoreceptors degeneration and then the RPE loss [10]. Perivascular thickening of the RNFL may support an additional vessel-based etiology for PPRCA rather than a RPE-based mechanism only. It has to be noted that chronic stages of $\mathrm{VKH}$ disease may present with several patterns of chorioretinal atrophy, including peripapillary atrophy, multiple nummular atrophic scars, irregular areas of atrophy and sectoral chorioretinal 
atrophy, which could have masqueraded as PPRCA [11]. In our case, the localized areas of retinal disturbance with normal chorioretinal paravenous zone in between lesions further suggest PPRCA diagnosis. Conversely, Huang et al. suggested that PPRCA following inflammatory retinal vein vasculopathy, resulting from various causes previously cited, are not real PPRCA and should be termed pseudo PPRCA [12]. In fact, a previous medical history is rarely found and appears to be a confounding rather than a causative factor [13].

\section{Conclusion}

In conclusion, we reported the first case of PPRCA occurring in a background of Vogt-Koyanagi-Harada disease. We highlighted multimodal imaging correspondence of this rare pathology and raised pathophysiologic hypothesis based on optical coherence tomography features.

\section{Abbreviations}

AF: Fundus autofluorescence; CME: Cystic macular edema; PPRCA: Pigmented paravenous retinochoroidal atrophy; RNFL: Retinal nerve fiber layer;

RPE: Retinal pigment epithelium; SD-OCT: Spectral domain optical coherence tomography; VKH: Vogt-Koyanagi-Harada

\section{Acknowledgements}

None.

\section{Authors' contribution}

PR drafted the work and performed acquisition and analysis of the data. PG, AC, DD designed the work and substantively revised it. All authors read and approved the final manuscript.

\section{Funding}

None.

\section{Availability of data and materials}

All data generated or analysed during the current study are included in this published article.

\section{Ethics approval and consent to participate}

not applicable. Ethical approval was not required as this manuscript presents a case study. Ethics board (Faculty La Timone, Marseille, France) approved this. It was performed in accordance with the tenets of the Declaration of Helsinki.

\section{Consent for publication}

The patient gave written permission for clinical details and images in this study. This report does not contain any personal information that could lead to the identification of the patient.

\section{Competing interests}

The authors declare that they have no competing interests.

Received: 24 May 2019 Accepted: 17 January 2020

Published online: 29 January 2020

\section{References}

1. Brown TH. Retino-choroiditis radiata. Br J Ophthalmol. 1937;21(12):645-8. https://doi.org/10.1136/bjo.21.12.645.

2. Choi JY, Sandberg MA, Berson EL. Natural course of ocular function in pigmented paravenous retinochoroidal atrophy. Am J Ophthalmol. 2006; 141(4):763-5. https://doi.org/10.1016/j.ajo.2005.11.009

3. McKay GJ, Clarke S, Davis JA, Simpson DAC, Silvestri G. Pigmented paravenous chorioretinal atrophy is associated with a mutation within the crumbs homolog 1 (CRB1) gene. Invest Ophthalmol Vis Sci. 2005:46(1):3228. https://doi.org/10.1167/iovs.04-0734.
4. Batioglu F, Atmaca LS, Atilla $H$, Arslanpençe A. Inflammatory pigmented paravenous retinochoroidal atrophy. Eye (Lond). 2002;16(1):81-4. https://doi. org/10.1038/sj.eye.6700021.

5. Obata R, Yanagi Y, Iriyama A, Tamaki Y. A familial case of pigmented paravenous retinochoroidal atrophy with asymmetrical fundus manifestations. Graefe's Arch Clin Exp Ophthalmo. 2006;244(7):874-7. https://doi.org/10.1007/s00417-005-0179-0

6. Shona OA, Islam F, Robson AG, Webster AR, Moore AT, Michaelides M. Pigmented paravenous chorioretinal atrophy: detailed clinical study of a large cohort. Retina. 2019;39(3):514-29. https://doi.org/10.1097/IAE. 0000000000001950

7. Aoki S, Inoue $T$, Kusakabe $M$, et al. Unilateral pigmented paravenous retinochoroidal atrophy with retinitis pigmentosa in the contralateral eye: a case report. Am J Ophthalmol Case Rep. 2017;8:14-7. https://doi.org/10. 1016/j.ajoc.2017.08.003.

8. Strong S, Liew G, Michaelides M. Retinitis pigmentosa-associated cystoid macular oedema: pathogenesis and avenues of intervention. $\mathrm{Br}$ Ophthalmol. 2017;101(1):31-7. https://doi.org/10.1136/bjophthalmol-2016309376

9. Ahmad M, Leisy H, Carr RE, Smith RT. A rare case of unifocal, unilateral pigmented paravenous retinochoroidal atrophy (PPRCA). Am J Ophthalmol Case Rep. 2016:4:41-4. https://doi.org/10.1016/j.ajoc.2016.08.009.

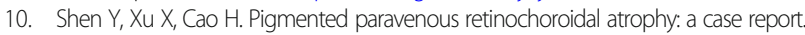
BMC Ophthalmol. 2018;18(1):136. https://doi.org/10.1186/s12886-018-0809-z

11. Vasconcelos-Santos DV, Sohn EH, Sadda S, Rao NA. Retinal pigment epithelial changes in chronic Vogt-Koyanagi-Harada disease: fundus autofluorescence and spectral domain optical coherence tomography findings. Retina. 2010;30(1):33-41. https://doi.org/10.1097/IAE. Ob013e3181c5970d

12. Huang H-B, Zhang Y-X. Pigmented paravenous retinochoroidal atrophy (review). Exp Ther Med. 2014;7(6):1439-45. https://doi.org/10.3892/etm.2014.1648.

13. Murray AT, Kirkby GR. Pigmented paravenous retinochoroidal atrophy: a literature review supported by a unique case and insight. Eye. 2000;14:7116. https://doi.org/10.1038/eye.2000.189.

\section{Publisher's Note}

Springer Nature remains neutral with regard to jurisdictional claims in published maps and institutional affiliations.
Ready to submit your research? Choose BMC and benefit from:
- fast, convenient online submission
- thorough peer review by experienced researchers in your field
- rapid publication on acceptance
- support for research data, including large and complex data types
- gold Open Access which fosters wider collaboration and increased citations
- maximum visibility for your research: over $100 \mathrm{M}$ website views per year
At BMC, research is always in progress.
Learn more biomedcentral.com/submission 